\title{
Antibody Response to SARS-CoV-2 is Associated with Long-term Clinical Outcome in Patients with COVID-19: a Longitudinal Study
}

\author{
Javier García-Abellán ${ }^{1}$ · Sergio Padilla ${ }^{1,2}$ - Marta Fernández-González ${ }^{1}$. José A. García ${ }^{3}$. Vanesa Agulló ${ }^{1}$.

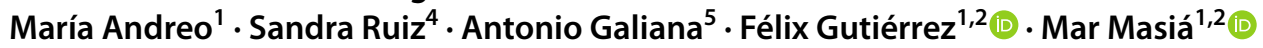

Received: 5 April 2021 / Accepted: 3 June 2021 / Published online: 17 July 2021

(c) The Author(s), under exclusive licence to Springer Science+Business Media, LLC, part of Springer Nature 2021

\begin{abstract}
Background The relationship of host immune response and viral replication with health outcomes in patients with COVID-19 remains to be defined. We aimed to characterize the medium and long-term clinical, virological, and serological outcomes after hospitalization for COVID-19, and to identify predictors of long-COVID.

Methods Prospective, longitudinal study conducted in COVID-19 patients confirmed by RT-PCR. Serial blood and nasopharyngeal samples (NPS) were obtained for measuring SARS-CoV-2 RNA and S-IgG/N-IgG antibodies during hospital stay, and at 1,2, and 6 months post-discharge. Genome sequencing was performed where appropriate. Patients filled out a COVID-19 symptom questionnaire (CSQ) at 2-month and 6-month visits, and those with highest scores were characterized. Results Of 146 patients (60\% male, median age 64 years) followed-up, 20.6\% required hospital readmission and 5.5\% died. At 2 months and 6 months, 9.6\% and 7.8\% patients, respectively, reported moderate/severe persistent symptoms. SARS-CoV-2 RT-PCR was positive in NPS in 11.8\% (median $\mathrm{Ct}=38$ ) and $3 \%$ (median $\mathrm{Ct}=36$ ) patients at 2 months and 6 months, respectively, but no reinfections were demonstrated. Antibody titers gradually waned, with seroreversion occurring at 6 months in $27(27.6 \%)$ patients for N-IgG and in $6(6 \%)$ for S-IgG. Adjusted 2-month predictors of the highest CSQ scores (OR [95\%CI]) were lower peak S-IgG (0.80 [0.66-0.94]) and higher WHO severity score (2.57 [1.20-5.86]); 6-month predictors were lower peak S-IgG (0.89 [0.79-0.99]) and female sex (2.41 [1.20-4.82]); no association was found with prolonged viral RNA shedding.

Conclusions Long-COVID is associated with weak anti-SARS-CoV-2 antibody response, severity of illness, and female gender. Late clinical events and persistent symptoms in the medium and long term occur in a significant proportion of patients hospitalized for COVID-19.
\end{abstract}

Keywords SARS-CoV-2 $\cdot$ COVID-19 $\cdot$ long-COVID $\cdot$ antibody response $\cdot$ viral shedding

Javier García-Abellán and Sergio Padilla contributed equally to the work and share first authorship. Félix Gutiérrez and Mar Masiá contributed equally to the work and share senior authorship.

\section{Félix Gutiérrez}

gutierrez_fel@gva.es

$\triangle$ Mar Masiá

marmasiac@gmail.com

1 Internal Medicine and Infectious Diseases Unit, Hospital General Universitario de Elche, Alicante, Spain

2 Clinical Medicine Department, Universidad Miguel Hernández de Elche, Alicante, Spain

\section{Introduction}

One year after the COVID-19 outbreak was first described [1], several questions about the disease remain to be answered. In contrast to the initial phases [2], long-term data following SARS-CoV-2 infection are limited. Dynamics of

3 Statistics, Operational Research Center, Universidad Miguel Hernández de Elche, Alicante, Spain

4 Section of Respiratory Medicine, Hospital General Universitario de Elche, Alicante, Spain

5 Microbiology Service, Hospital General Universitario de Elche, Alicante, Spain 
SARS-CoV-2 in the long term, including the persistence of viral shedding, the incidence of late viral rebounds, or reinfections, and their relationship with the clinical evolution of patients have not been defined. From the immunological perspective, another relevant question refers to the durability of the antibody response, and the impact of the intensity and duration of response on patients' outcomes. In a significant proportion of patients, symptoms persist after hospital discharge for more than 2 months, which has been defined as long-COVID [3-5]. In addition to a more comprehensive characterization of the syndrome, the pathogenic mechanisms involved, including the role of viral shedding and the antibody kinetics, need to be determined. Acute respiratory distress induced by SARS-CoV-2 has been associated with persistent inflammation and pro-coagulation [6], which might potentially contribute to incomplete recovery, but the kinetics of inflammation and coagulation biomarkers after prolonged follow-up have not been disclosed.

We have longitudinally followed a cohort of patients hospitalized with COVID-19 who have been thoroughly investigated over a 6-month period after discharge. Our objective was to characterize the medium and long-term clinical, virological, and immunological outcomes, and to identify evolutionary trajectories and predictors of long-COVID.

\section{Methods}

\section{Study Design, Patients, and Study Procedures}

This prospective, longitudinal study was carried out at Hospital General Universitario de Elche, Spain. All patients admitted for COVID-19 between March 10 and June 30, 2020, were included in the analysis and were followed-up until December 31, 2020, the administrative censoring date of the study dataset. Cases included in the study were microbiologically confirmed through real-time polymerase chain reaction (RT-PCR) from nasopharyngeal swab samples in most cases and from fecal samples in 8 .

Hospitalized COVID-19 patients were managed according to a predefined local protocol that included the diagnostic and therapeutic procedures during hospital stay [7]. This protocol consisted on the standardized collection of clinical variables and serial blood and nasopharyngeal sampling, obtained at different time-points during hospital stay for biochemical and sero-virological measurements.

Once discharged, patients' follow-up was centralized at the Infectious Diseases Unit Outpatients' clinic. Like other authors [8], we have focused on two periods of the post-acute COVID-19 timeline, the ongoing symptomatic COVID-19, which includes symptoms and abnormalities present from 4 to 12 weeks beyond acute COVID-19, and the post-COVID-19 syndrome, which includes symptoms and abnormalities persisting or present beyond 12 weeks of the onset of acute COVID-19. Accordingly, face-to-face visits were scheduled in the ongoing symptomatic COVID-19 period ( 1 and 2 months visits, herein also "mid-term") and in the post-COVID-19 period (6-month visits, herein also "long term") after discharge. On each visit, blood and nasopharyngeal samples were obtained for biochemical and serovirological measurements (Figure S-1). Phone and face-toface visits not foreseen in the protocol were also appointed at the patients' request.

At 2-month and 6-month visits, patients were offered to fill out a self-administered, self-rated COVID-19 symptom questionnaire (CSQ, Annex 1) in which they had to grade 11 items using a 10-point increasing intensity scale $(0=$ absence of the symptom and $10=$ the maximum perceived intensity of the symptom). To characterize the persistence of symptoms, and as a test for robustness, we defined three different categories of the CSQ score: a score of at least one point (any symptom-CSQ); a score equal or more than the median (median-CSQ), and a score above the third quartile (highest CSQ score) in any of the CSQ items. Scores were also classified into mild ( $1-4$ points), moderate ( $5-7$ points), and severe (8-10 points) categories.

In patients who missed scheduled appointments, an attempt was made to contact them by phone and their electronic medical records were carefully scrutinized to ensure the vital status of the patients. Hospital readmissions occurring during the 6-month follow-up period were reviewed and recorded in the dataset.

\section{SARS-CoV-2 RNA and Antibody Measurements}

RT-PCR analysis for SARS-CoV-2 was performed by means of a commercially available kit (AllplexTM 2019nCoV Assay, Seegene, Seoul, Korea) which targeted the E, RdRP, and N genes. Suspected SARS-CoV-2 reinfection was defined according to the CDC criteria [9]. Briefly, subjects with detected SARS-CoV-2 RNA more than 90 days after the first detection of SARS-CoV-2 RNA, whether or not symptoms were present, and if detected by RT-PCR, only considering if $\mathrm{Ct}$ value was less than 33 or if $\mathrm{Ct}$ value was unavailable. Genome sequencing of SARS-CoV-2 was performed on NPS samples following ARTIC amplicon sequencing protocol for MinIon version V3 (see Supplementary material for full description). IgG antibody plasma levels against the SARS-CoV-2 internal nucleocapsid (N) protein (N-IgG) (Anti-SARS-CoV-2-NCP IgG ELISA, Euroimmun, Lubeck, Germany) and surface $\mathrm{S} 1$ domain of the spike protein (S-IgG) (Anti-SARS-CoV-2 IgG ELISA, Euroimmun, Lubeck, Germany) were measured using commercial semi-quantitative EIA kits in an automated instrument (Dynex DS2® ELISA system). More procedures details can be found elsewhere [7]. 


\section{Statistical Analyses}

Continuous variables are expressed as median \pm 25 th and 75 th percentiles $(\mathrm{Q} 1, \mathrm{Q} 3)$, and categorical variables as percentages. Wilcoxon or Student's $t$ test were used to compare continuous variables, and the chi-square or Fisher's exact test for categorical variables comparison among patients with and without persistent symptoms. To compare the curves of viral load, antibody levels, and biomarkers between groups, generalized additive mixed models were used. Interpolations in the graphs were carried out with cubic splines. Covariates with a $p$-value $<0.05$ in the crude comparison between groups and clinical relevant variables were included in multivariate analyses. Binomial logistic regression models were used to identify predictors of persistence of symptoms at 2 and 6 months. A receiver operating characteristic (ROC) curve analysis was performed to find the most discriminative serum level of anti-SARS-CoV-2 antibodies predicting persistence of symptoms. Statistical analysis was performed using R-project version 3.6.2.

\section{Results}

\section{Patient's Characteristics}

A total of 162 patients were hospitalized with SARS-CoV-2 infection, 23 (14\%) were admitted to the ICU and 12 (7.4\%) died during hospital stay. After hospital discharge, 4 patients were lost to follow-up. Flow chart of patients is shown in Figure S-2. One hundred and four and 116 patients completed the SCQ at 2 and 6 months, respectively.

Baseline characteristics of the 146 included patients are shown in Table S-1. Median age was 64 years, 88 (60.3\%) were male, and $72.6 \%$ had coexisting comorbid diseases. Clinical status on admission and therapy administered during hospital stay are detailed in Table S-1.

\section{Clinical and Biological Outcomes}

During follow-up, 30 patients (20\%) were readmitted to hospital (Table S-2). The most frequent reasons for hospital readmission were underlying disease exacerbation (23 events in 13 patients, $8.9 \%$ ), bacterial infection (12 in 5 patients, $3.4 \%$ ), thrombohemorrhagic events (9 in $9,6.1 \%$ ), and persistent COVID-19 symptoms (8 in 6, 4.1\%) patients. Eight (5.5\%) patients died. Detailed information of causes of inhospital and after discharge deaths are shown in Table S-2.

Persistent symptoms of moderate or severe intensity at the 2-month visit were observed in $9.6 \%, 7.4 \%$, and $2.9 \%$ patients for general, gastrointestinal, and respiratory symptoms, respectively, and in $7.8 \%, 4.3 \%$, and $1.0 \%$ patients, respectively, at the 6-month visit (Fig. 1).
Serum inflammatory biomarkers showed an initially steep and later flatter substantial decrease during follow-up, followed by stabilization or non-significant ulterior increase. The most prominent initial decrease was observed with C-reactive protein (CRP). Temporal changes in the levels of several biomarkers throughout the study period are shown in Fig. 1.

\section{Virological Outcomes}

SARS-CoV-2 RNA shedding lasted a median (Q1-Q3) of 13 (2.2-33.8) days in those with the last RT-PCR test negative (Table S-1), resulting in a proportion of patients who tested negative at 2-month and 6-month follow-up visits of $88 \%$ and $97 \%$, respectively. Viral RNA shedding of low intensity was detected in some patients in subsequent nasopharyngeal tests beyond the acute phase of the disease. Thus, 40/146 (27\%; median [Q1-Q3] Ct $=34$ [31-37]), 15/127 (11.8\%; median $\mathrm{Ct}=38$ [37.25-39]), and 4/134 (3.0\%; median $\mathrm{Ct}=36$ [36-36]) individuals tested positive at month 1,2 , and 6 visits, respectively. SARS-CoV-2 RT-PCR results during follow-up are displayed in Fig. 2a. Although none of the four patients with positive SARS-CoV-2 RNA at month 6 met the CDC criteria for suspected reinfection [9], sequencing could be performed in three of them for whom paired stored samples were available. Patients' age ranged from 44 to 73 years, two were men and two had subjacent comorbidities (breast cancer in remission in one patient and epilepsy and mental retardation in another). Two patients received immunomodulatory therapy (high-dose corticosteroids or interferon- $\beta-1 b$ ) during COVID-19 hospital admission. The same clade 20B (lineage B.1.1) was present in all cases (see Figure $\mathrm{S}-3$ for phylogenetic assignment of the samples and current notable variants). In two patients, the clade showed the same hallmark single nucleotide variants (individuals \#88 and \#95, Table S-3), and in the third patient, two new mutations were detected in the most recent sample (a K374R substitution in the $\mathrm{N}$ gene and an A222V substitution in the $\mathrm{S}$ gene), probably developed due to persistent infection.

\section{Serological Outcomes}

Median [Q1-Q3] time from illness onset to seropositivity was 12 (8-15) days. Peak S-IgG was significantly higher compared to $\mathrm{N}-\mathrm{IgG}$ (median 5.9 vs. 4.1 absorbance/cutoff $[\mathrm{S} / \mathrm{CO}] ; p<0.001$, respectively) (Table $\mathrm{S}-1$ ). Figure $2 \mathrm{~b}$ shows $\mathrm{S}-\mathrm{IgG}$ and $\mathrm{N}-\mathrm{IgG} \mathrm{S} / \mathrm{CO}$ values of all determinations of the study patients over time. 
A

General symptoms*

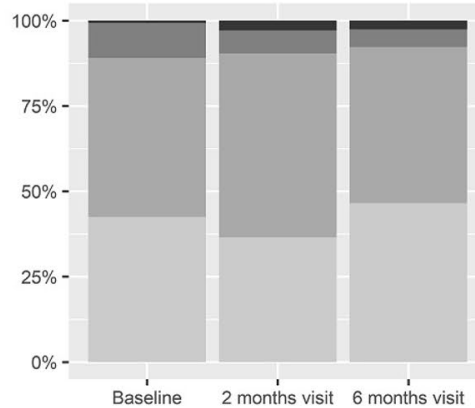

Respiratory symptoms*

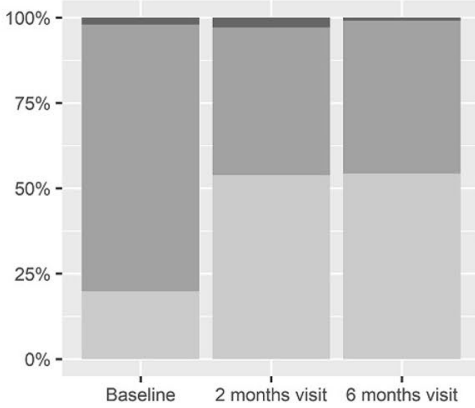

No symptoms (0)
Gastrointestinal symptoms*

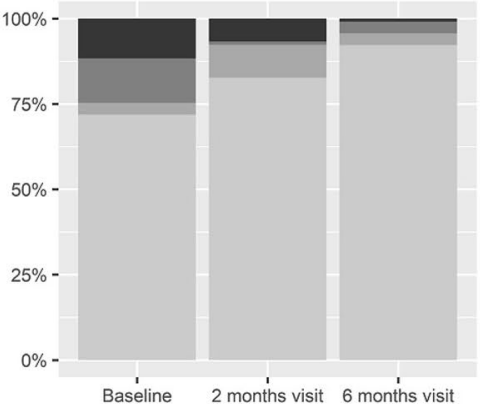

Severe (8-10)

B

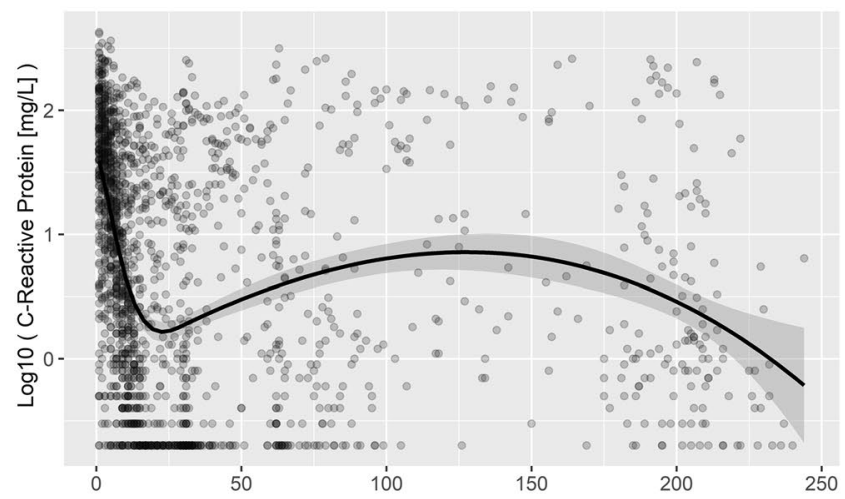

D

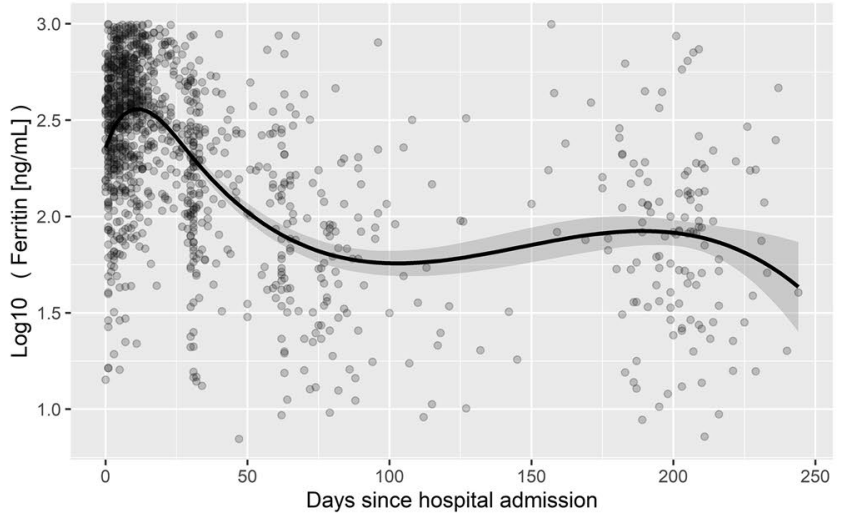

Fig. 1 Temporal changes in symptom scores and serum levels of biomarkers during follow-up. Panel A shows the scores of self-reported general, respiratory, and gastrointestinal symptoms included in the COVID-19 symptoms questionnaire at baseline, 2 months and 6 months. Panels B, C, D, E represent the temporal changes in the serum levels of C-reactive protein (panel B), interleukin-6 (panel C), ferritin (panel D), and D-dimer (panel E) since COVID-19 diagnosis. Panel A: General symptoms include fatigue, myalgia, sweating, headache; respiratory symptoms include cough, difficulty breathing, nasal congestion, sore throat, anosmia; gastrointestinal symptoms include

Antibody titers gradually waned, and $29(28.7 \%)$ patients became seronegative for either N-IgG or S-IgG during follow-up: 23 for N-IgG alone, 2 for S-IgG alone, and 4
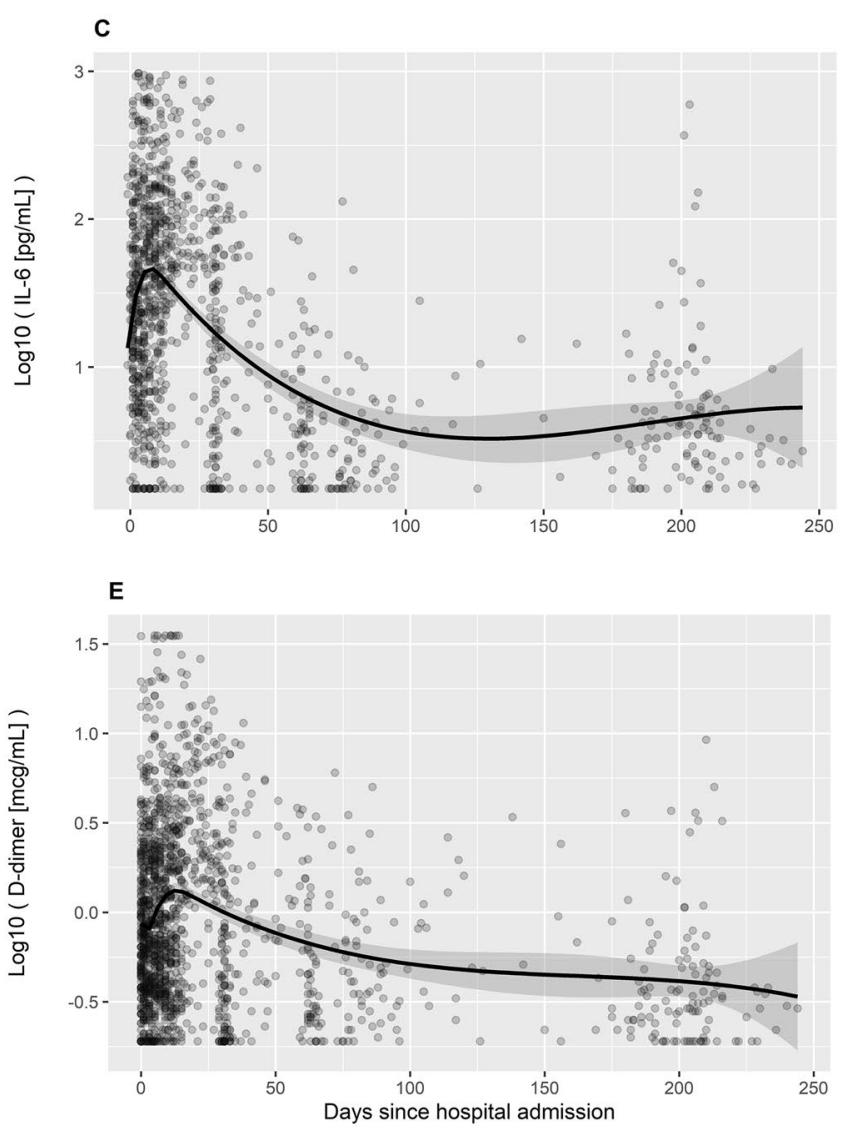

diarrhea, vomiting, abdominal pain. "Pearson's Chi-squared test $P$ value $<0.05$. Panels $\mathbf{B}, \mathbf{C}, \mathbf{D}, \mathbf{E}$ : Each dot represents a serum biomarker value in an individual subject since hospital admission, with interpolation line and $90 \%$ confidence interval. Biomarkers normal ranges: C-reactive protein, below $5 \mathrm{mg} / \mathrm{L}$; IL-6, below $7 \mathrm{pg} / \mathrm{mL}$; ferritin, normal ranges values depend on patient's sex and age (17-464 $\eta \mathrm{r} / \mathrm{mL}$ for men older than 20 years and 6-137 $\eta \mathrm{g} / \mathrm{mL}$ and $11-264$ $\eta \mathrm{g} / \mathrm{mL}$ for women between 20 and 50 and older than 50 years old, respectively); D-dimer, below $0.5 \mu \mathrm{g} / \mathrm{mL}$. IL-6, interleukin-6

for both N-IgG and S-IgG. The majority of cases of seroreversion were observed at the 6-month visit (25/29 [86.2\%] patients). 
A

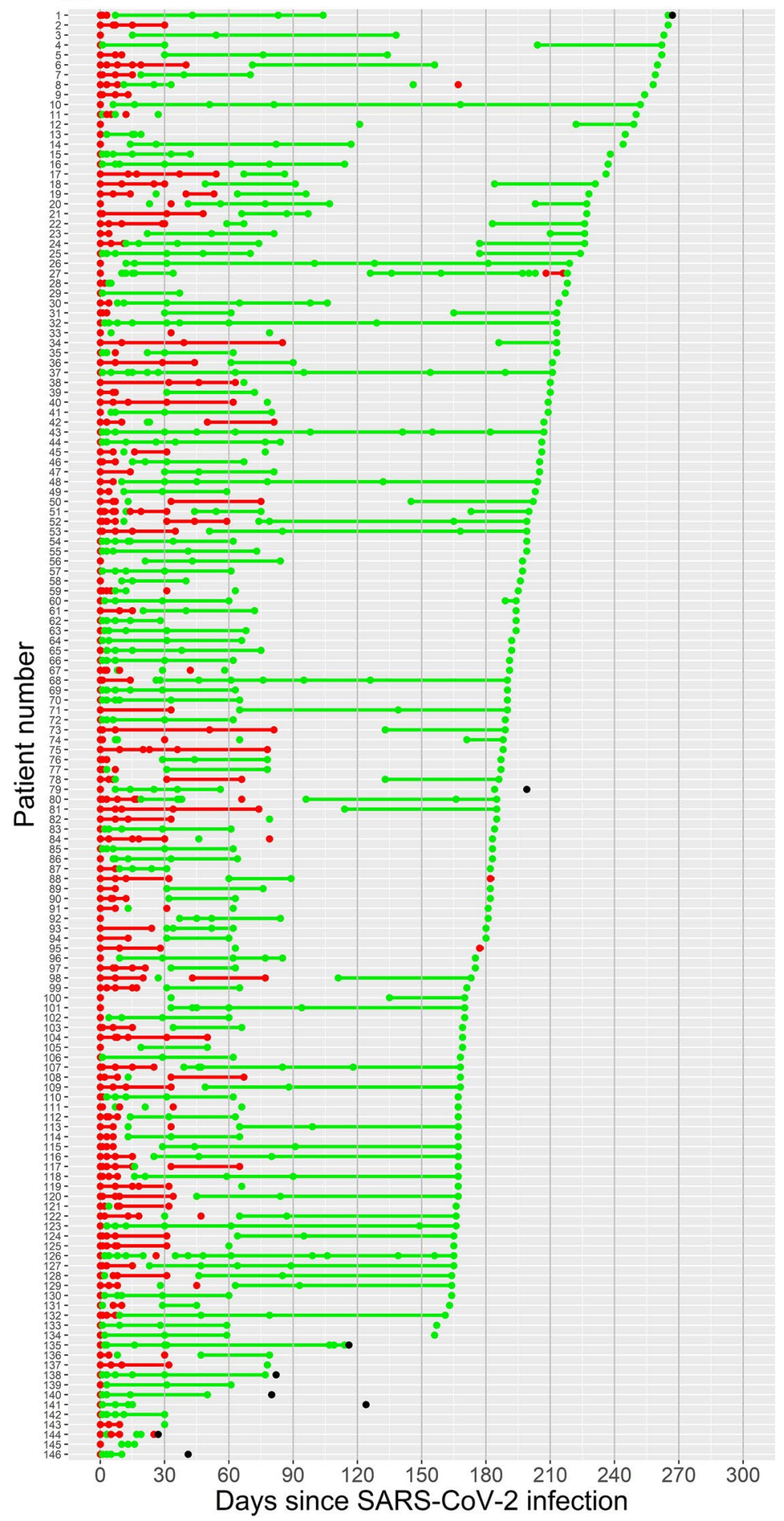

B

- $\log [S-\lg G(S / C O)]+\log [N-\lg G(S / C O)]$

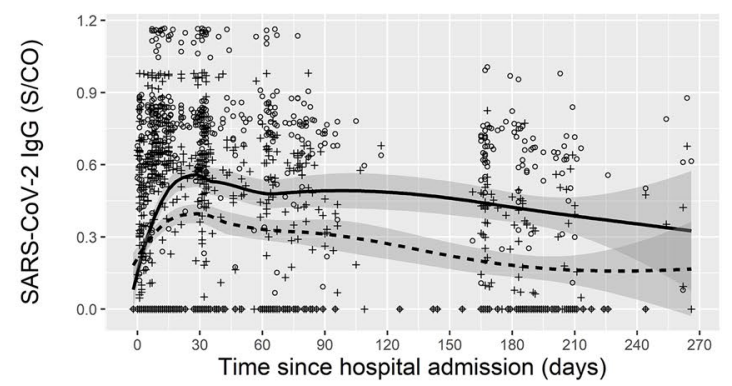

Fig. 2 SARS-CoV-2 sero-virological changes during follow-up. Panel A: SARS-CoV-2 RT-PCR test results during follow-up in the entire cohort. Panel B: Temporal changes in anti-SARS-CoV-2 surface S1 domain of the spike protein and nucleocapsid protein $\mathrm{IgG}$ antibodies. Panel A: Each horizontal level represents the RT-PCR SARS-CoV-2 results for each patient in the cohort throughout follow-up, since the first positive result to the last available one. Red dots represent positive results and green dots negative results. Black dots mark those patients who died and time of death since the first positive SARS-CoV-2 RT-PCR result. Colored lines join two consecutive dots in the same patient with the same result and less than 90 days between dots. Patient \#143 moved to another country after discharge. Patients \#139, \#142, and \#145 could only be reached by phone. Patients \#136 and \#137 refused to have a new nasopharyngeal sample taken but agreed to continue with the clinical follow-up. RT-PCR, reverse transcriptase-polymerase chain reaction. Panel B: Each dot represents an S-IgG (continuous line and circle symbols) or N-IgG (dashed line and plus symbols) value in an individual subject, with interpolation line and $90 \%$ confidence interval. S/CO, absorbance/cut-off; S-IgG, S-IgG antibody against the SARS-CoV-2 surface $\mathrm{S} 1$ domain of the spike protein; N-IgG, antibody against the SARS-CoV-2 internal nucleocapsid protein. Positive $\mathrm{S} / \mathrm{CO}>1.1$ 


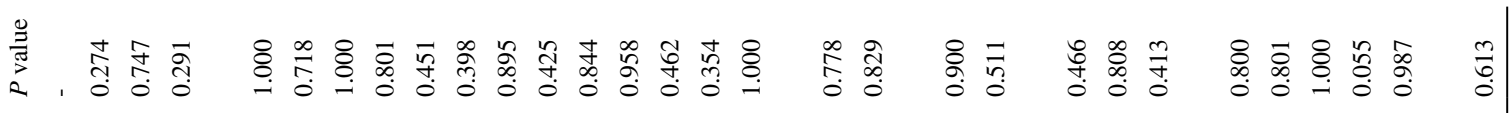

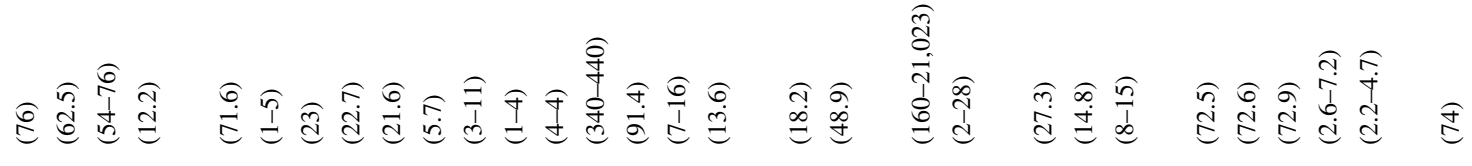

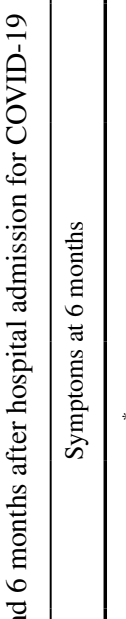

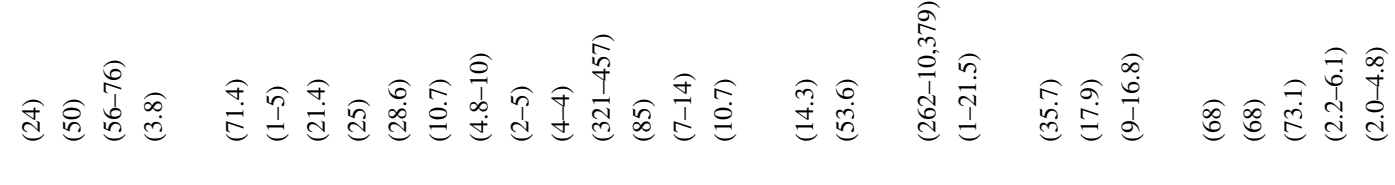

乐进士品-

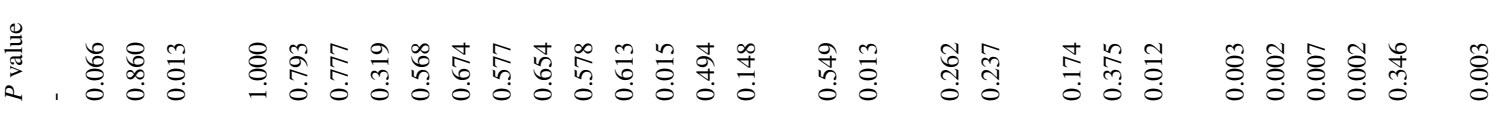

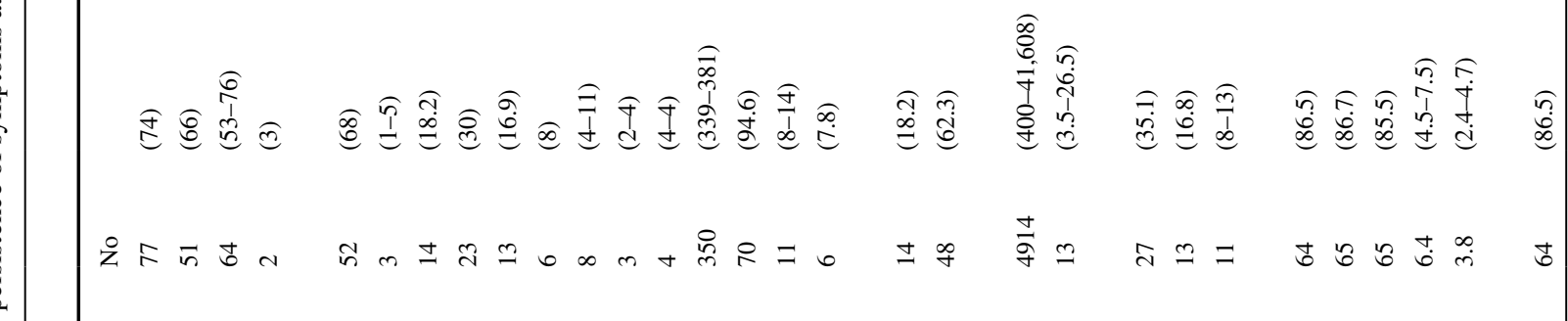

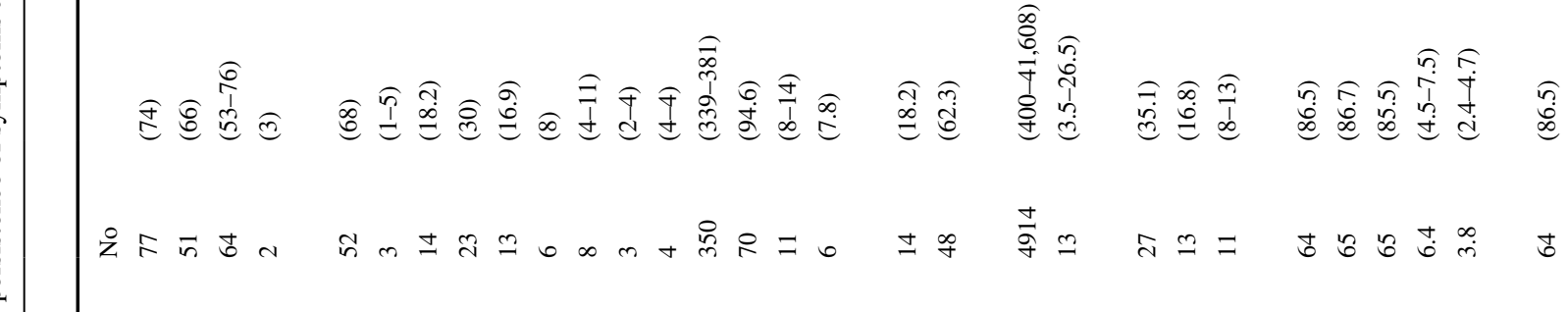

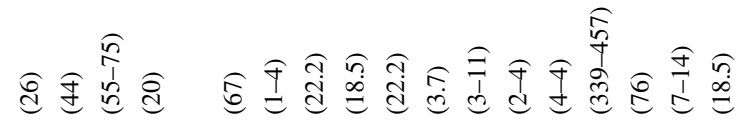

舟 in

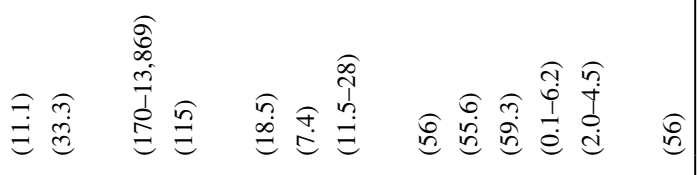

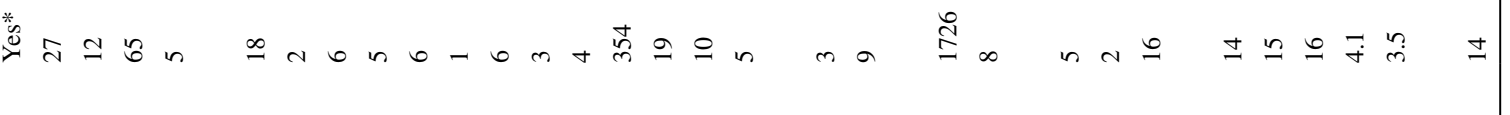

$$
\text { (1) }
$$




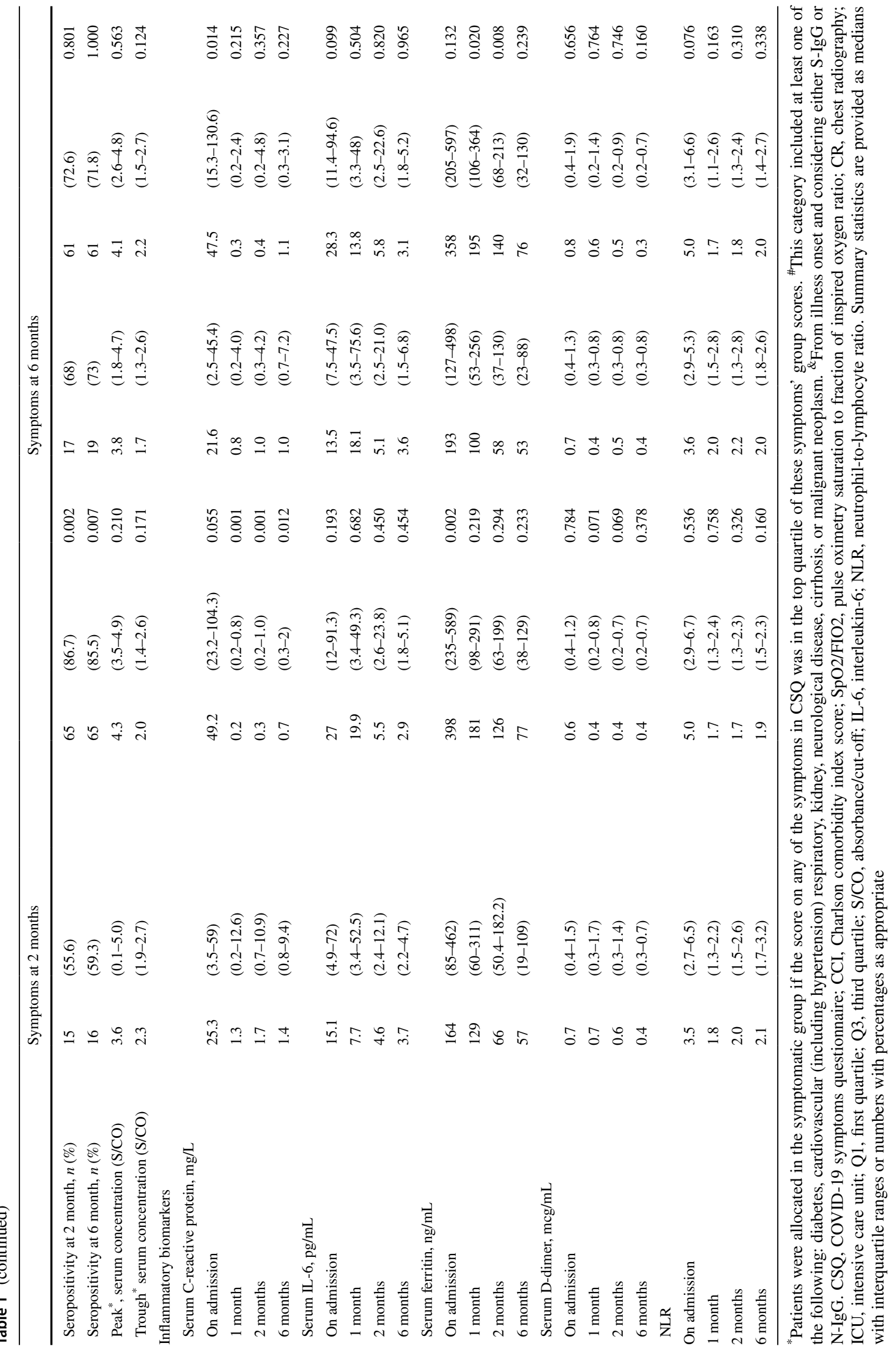



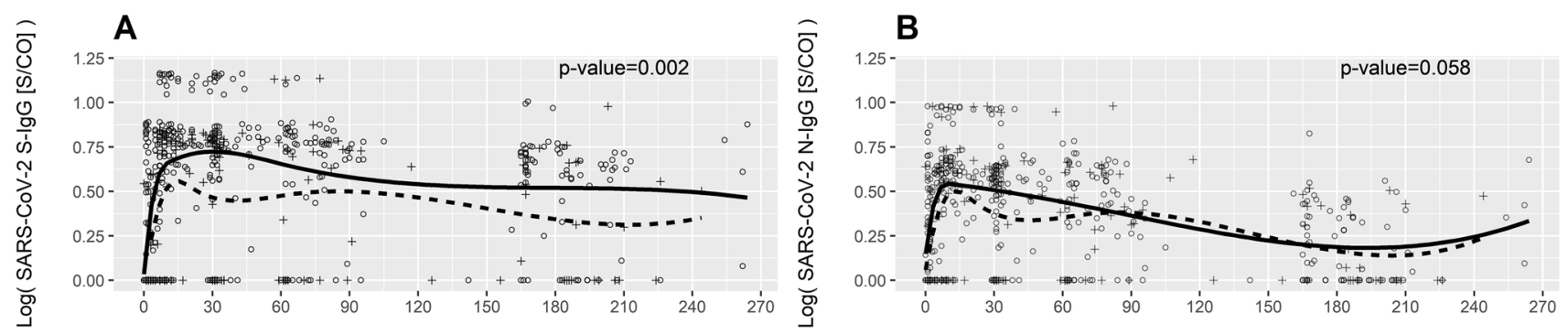

C
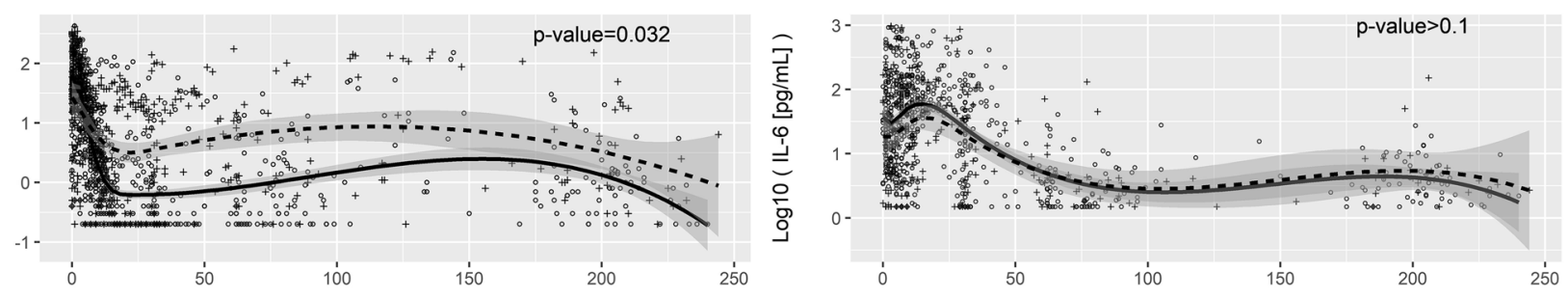

E
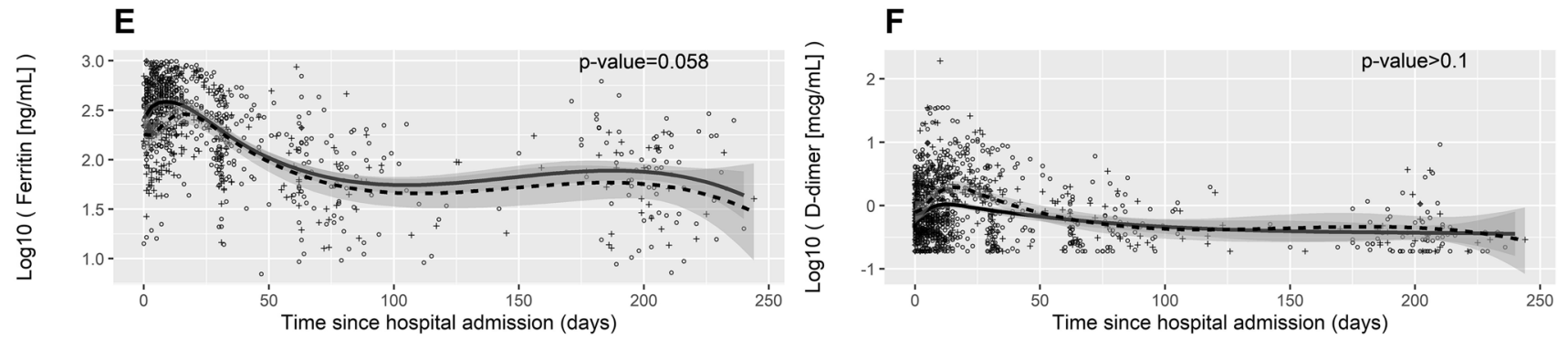

Fig. 3 Temporal changes in SARS-CoV-2 IgG antibodies and serum inflammatory biomarkers during follow-up by symptomatic status. Serum levels of S-IgG (panel A), N-IgG (panel B), C-reactive protein (panel C), interleukin-6 (panel D), ferritin (panel E), and D-dimer (panel F) during follow-up. Dashed lines and plus symbols represent individuals with the highest scores in COVID-19 symptoms questionnaire; continuous lines and circle symbols represent all

\section{Characterization and Predictors of Short-term Persistence of Symptoms}

A significant number (76 [73\%]) of patients scored any symptom-CSQ, being fatigue (54.8\%), myalgia (30.8\%), dyspnoea (26.9\%), and cough (25\%) the most frequent. The most frequent symptoms reported by patients with the highest CSQ scores were fatigue (12.5\%), myalgia (7.6\%), and dyspnoea (6.7\%).

Table 1 shows the characteristics of the patients with the highest CSQ scores at 2 months. In the univariate analysis, they were more frequently active smokers $(p=0.013)$, had more frequently bilateral lung infiltrates $(p=0.015)$, exhibited at hospital admission lower baseline ferritin levels $(p=0.002)$, a trend to lower CRP $(p=0.055)$, and had received less frequently tocilizumab $(p=0.013)$ during the acute episode of COVID-19.

No differences were found between groups in the $\mathrm{Ct}$ values on admission (E-gene, 29.1 [26.5-32.8] vs. 29.7

other patients filling the questionnaire. S-IgG, IgG antibody against the SARS-CoV-2 surface $\mathrm{S} 1$ domain of the spike protein; $\mathrm{N}-\mathrm{IgG}$, IgG antibody against the SARS-CoV-2 internal nucleocapsid protein; S/ $\mathrm{CO}$, absorbance/cut-off. $P$ value for the comparison between groups according to the score in COVID-19 symptoms questionnaire. Positive $\mathrm{S} / \mathrm{CO}>1.1$

[26.2-34.3]; $p=0.909)$, time to first negative RT-PCR results ( 8 vs. 13 days; $P=0.237$ ), the proportion of individuals who tested positive beyond 1 -month $(19 \%$ vs. $35 \% ; p=0.174)$ and 2 -month $(7.4 \%$ vs. $16.8 \% ; p=0.375)$ visits (Table 1$)$, and in the $\mathrm{Ct}$ values at 1-month (E-gene, 35 [33.0-36.0] vs. 36 [31.5-37.5]; $p=0.754$ ) or 2-month (E-gene, 38.5 [38.2-38.8] vs. 38.0 [37.0-39.0]; $p=1.000)$ visits.

There was a weaker antibody response against SARSCoV-2 in the highest CSQ scores group, consisting of a longer time to either S-IgG or N-IgG seroconversion from illness onset (median 16 vs. 11 days; $p=0.012$ ), a flatter slope $(+0.4[0-0.5]$ vs. $+1.0[0.5-3.2]$ change in $\mathrm{S} / \mathrm{CO}$ per day; $p<0.001)$ until reaching peak levels, and lower peak $\mathrm{S}-\mathrm{IgG}$ value (4.1 vs. $6.4 \mathrm{~S} / \mathrm{CO} ; p=0.002)$ (Table 1 and Fig. 3). Accordingly, fewer patients within the highest CSQ scores showed seroconversion at 2 months for S-IgG and for $\mathrm{N}-\mathrm{IgG}(55.6 \%$ vs. $86.7 \% ; p=0.002)$.

Receiver operating characteristic (ROC) curve analysis showed that a SARS-CoV-2 S-IgG value below 5.4 S/CO at 
Fig. 4 Predictors of the highest scores in COVID-19 symptoms questionnaire in multivariate regression logistic model at 2-month and 6-month followup. Panel A represents 2-month, and panel $\mathbf{B}$ 6-month follow-up. WHO, World Health Organization; S-IgG, IgG antibody against the SARS-CoV-2 surface $\mathrm{S} 1$ domain of the spike protein; S/CO, absorbance/cut-off; RT-PCR, reverse transcriptasepolymerase chain reaction. ${ }^{*} P$ value $<0.05$
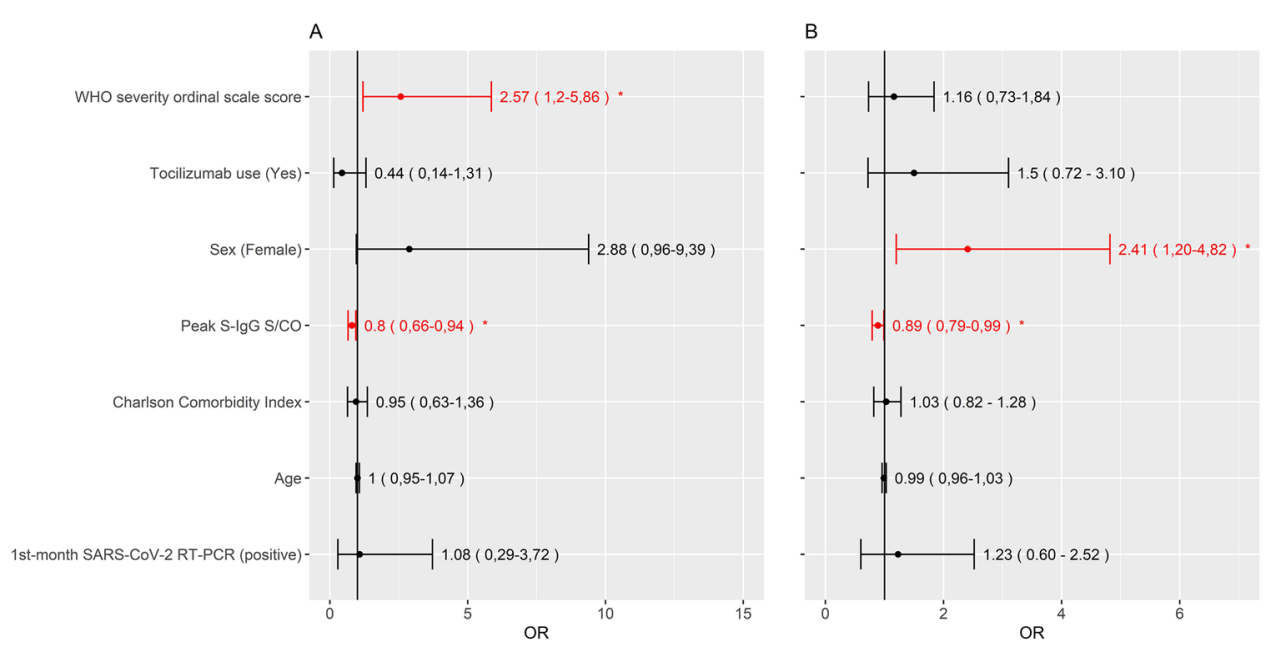

1-month post-discharge predicted the highest CSQ scores at 2 months with an AUC [CI 95\%] of 0.66 [0.54-0.79] (Figure S-4).

A differential profile in the dynamics of CRP levels was observed according to group, with a trend to lower levels at baseline in individuals with highest CSQ scores, and subsequent inversion in the trend at 1-month and 2-month visits (Fig. 3 and Table 1). Baseline ferritin levels were higher in the group with highest CSQ scores. No differences were found in other inflammatory biomarkers among groups.

In the multivariate logistic regression analysis including age, sex, Charlson comorbidity index, WHO severity ordinal scale score, peak S-IgG values, testing positive for SARS-CoV-2 RT-PCR at 1-month visit and tocilizumab use, we found that a lower peak in SARS-CoV-2 $\mathrm{S}-\mathrm{IgG}$ value (OR [95\% CI] 0.80 [0.66-0.94]), and a higher WHO severity scale score (2.57 [1.20-5.86] per point increase) were independent predictors of the highest CSQ scores at 2 months after discharge (Fig. 4a). Sensitivity analyses with the outcomes median-CSQ score or any symptom-CSQ showed similar trends (Figure S-5a-b).

\section{Characterization and Predictors of Long-term Persistence of Symptoms}

One hundred and sixteen individuals, including 85 who also had completed the survey at 2 months, voluntarily agreed to fill out the CSQ at the 6-month visit. The most frequent symptoms reported by patients with the highest CSQ scores were fatigue (10.3\%), myalgia (6.9\%), dyspnoea (4.3\%), cough $(4.3 \%)$, and nasal congestion $(4.3 \%)$.
Patients with the highest CSQ scores showed lower CRP levels on admission and a trend to lower peak S-IgG value (Table 1).

In the multivariate logistic regression analysis including the same variables as in 2-month visit, a lower peak in SARSCoV-2 S-IgG (OR [95\% CI] 0.89 [0.79-0.99]) and female sex (2.41 [1.20-4.82] were predictors of the highest CSQ scores at the 6-month visit (Fig. 4b). Sensitivity analyses with medianCSQ score and any symptom-CSQ showed similar trends (Figure S-5 c-d).

\section{Discussion}

In this closely followed-up cohort evaluating the clinical, serological, and virological outcomes of patients admitted to hospital for COVID-19 during a 6-month period, we observed a high frequency of clinical events following hospital discharge. Most of these events were related to patients' underlying comorbidities, secondary bacterial infections, and recurrence or complications associated with COVID-19, including thrombohemorrhagic events. A proportion of patients reported persistent symptoms in the medium ( 2 month) and half of them in the long term (6 month). Chronic persistence of symptoms was associated with a distinct serological, inflammatory, and clinical profile, which differed according to duration of symptoms. Patients with the highest CSQ scores both in the mid-term and longterm exhibited a weaker initial antibody response and lower inflammation during the acute phase of the disease. Lasting symptoms in the mid-term were associated with persistent inflammation at months 1 and 2 after 
discharge and higher WHO severity score. Female sex predicted long-term complaints.

Patients included in this longitudinal study were comprehensively monitored. From the virological perspective, after a median duration of viral shedding of around 2 weeks, we found evidence of prolonged viral replication, as shown by persistent RT-PCR test positivity (or re-positivity) 2 months after discharge in $12 \%$ of the patients, but only four cases had a RT-PCR positive in the very late phases of follow-up. Interestingly, most patients with late RT-PCR positivity had high $\mathrm{Ct}$ values, and none of them fulfilled the $\mathrm{CDC}$ criteria for potential reinfection [9]. Moreover, genotypic sequencing confirmed the same clade in all analyzed samples, suggesting that they represented persistent infection/reactivation.

S-IgG antibodies reached higher titers, followed by a gradual decline of both S-IgG and N-IgG. Seroreversion was observed for $\mathrm{N}$-IgG titers in more than one fourth of patients at 6 months after discharge, but it was infrequent with the potentially neutralizing S-IgG antibodies, which implies a durable antibody response in patients with severe COVID requiring hospitalization, in contrast to the rapid waning of antibodies reported in patients with mild COVID [10-12]. Severity of disease has actually been associated with the magnitude and duration of the antibody response [13].

While long-COVID is increasingly being described after acute infection, the pathogenesis and duration of this multifaceted syndrome remain unknown. Data about longCOVID with such a lengthy follow-up are very scarce [14]. Our data suggest an involvement of the antibody response in the occurrence of long-COVID. The highest CSQ scores in the medium and long term were associated with a lower peak of S-IgG, and in patients with mid-term symptoms a delayed antibody response was additionally observed, the latter also showing to be a predictor of the highest CSQ scores in multivariate analysis (data not shown). Experimental studies have demonstrated that, in addition to T-cell response, IgG, and neutralizing antibodies are crucial to control viral infections $[15,16]$. Besides the antimicrobial activity, antibodies contribute to modulate the inflammatory response via the Fc-gamma receptor, toll-like receptors, and activation of the complement, all of them inducing the secretion or repression of various pro-inflammatory and anti-inflammatory mediators [17-20]. Both the antiviral and the immunomodulatory effects of antibodies might have been involved in the enhanced recovery of patients with higher antibody levels in our cohort. The protective effects of immunoglobulins are actually the basis for the use of convalescent plasma [21,22] or neutralizing monoclonal antibodies against SARS-CoV-2 spike protein [23] to treat patients with acute COVID-19. Unfortunately, no patient in our study was treated with convalescent plasma or neutralizing monoclonal antibodies to test this hypothesis. Monoclonal antibodies or boosting the antibody response with vaccination might be potential strategies to prevent long-COVID in patients with low antibody titers. This hypothesis warrants further research.

Post-COVID syndrome was associated with additional distinctive innate and adaptive immune traits, consisting of a weaker initial inflammatory response, as shown by lower baseline levels of CRP and ferritin. This is aligned with the poorer antibody response observed in this subset of patients, and supports the correlation described between antibody levels and inflammation biomarkers [24]. A less intense immune/inflammatory response may lead to impaired or incomplete control of viral replication and eventually greater long-term sequelae and symptoms. On the other hand, potent innate and adaptive immune responses during acute infection might potentially lead to a more efficient control of illness and lower residual inflammation and incidence of subsequent sequelae. Interestingly, patients with mid-term lasting symptoms showed persistent residual inflammation. Elevated markers of inflammation and autoimmunity have been reported in post-infectious syndromes linked with Chikungunya or Epstein-Barr viruses, or in myalgic encephalomyelitis [25-27].

Besides the immune response, demographic and clinical factors were found to be predictors of long-COVID. Long-term persistent symptoms were associated with female sex, a finding also supported by other studies [14, 28]. Higher severity of disease showed to be a predictor of mid-term persistent symptoms. Severe disease and the hyperinflammatory response occurring in the late phase would be expected to induce greater organ injury, which might be associated with higher incidence of sequelae, as supported by the higher prevalence of persistent symptoms found in patients with COVID-19 admitted to the ICU $[14,29]$. Although post-discharge complaints have also been described in patients with milder forms of COVID, critically ill patients have more frequently shown subjacent structural causes contributing to explain the symptoms [30], and up to $80 \%$ of them develop physical, cognitive, or mental health impairments that persist beyond hospital discharge [31]. Differences in patients' characteristics and in the intensity of symptoms might have contributed to the higher degree of reversibility potentially occurring in our patients, in whom severity of disease did not show to be a predictor of long-term high-score symptoms compared to the results of Huang et al. [14].

The sample size is a limitation of the study. Long-COVID is a heterogeneous syndrome and the associations found might differ according to the nature of the symptoms analyzed. We focused on subjects reporting the highest scores in the questionnaire to identify those symptoms that were more likely to be meaningful to patients and avoid overrepresentation of long-COVID cases. However, sensitivity analyses including patients with any symptom, or those with 
a score above the median, showed similar results. For some of the figures, the interpolation lines in certain intervals were developed with relatively low number of data. Strengths are the longitudinal design with consecutive sampling, close monitoring, and thorough investigations conducted in the patients.

In conclusion, long-term follow-up of patients hospitalized for COVID shows a high frequency of clinical events after hospitalization, a durable antibody response of S-IgG, and frequent RT-PCR test positivity/re-positivity occurring beyond 2 months after acute infection, but with no evidence of reinfection. Persistent symptoms are common in the medium and long term. Gender, severity of illness and the immune response are associated with long-COVID, but with different implication according to the temporality of symptoms. The antibody response predicts both mid-term and long-term clinical outcomes, and consequently the use of monoclonal antibodies or boosting the antibody response with vaccination might be potential strategies to prevent long-COVID.

\section{Data Availability of Data, Code, and Material}

Data and statistical code are available from the corresponding author upon reasonable request.

Supplementary Information The online version contains supplementary material available at https://doi.org/10.1007/s10875-021-01083-7.

Author Contributions MM and FG conceived the study. MM, FG, JGA, and SP designed the study. JGA and SP collected and were responsible for the data. JAG performed the statistical analysis. MM, FG, JGA, SP, MA, and SR participated in patient care, investigation, and data collection. MFG, VA, AG performed the microbiology analyses. MM, FG, JGA, and SP wrote the first draft of the manuscript. All authors drafted the manuscript for important intellectual content, contributed to the revision of the final version of the manuscript, and approved the final version submitted.

Funding FG, MM, SP, and MFG were supported by RD16/0025/0038 project as a part of the Plan Nacional Research + Development + Innovation $(\mathrm{R}+\mathrm{D}+\mathrm{I})$ and cofinanced by Instituto de Salud Carlos IIISubdirección General de Evaluación y Fondo Europeo de Desarrollo Regional; Instituto de Salud Carlos III (Fondo de Investigaciones Sanitarias [grant number PI16/01740; PI18/01861; CM19/00160, CM20/00066, COV20/00005]). AG was supported by COV20/00156.

\section{Declarations}

Ethics Approval Approval was granted by the ethics committee of Hospital General Universitario de Elche (PI 46/2020). The procedures used in this study adhere to the principles of the Declaration of Helsinki.

Consent to Participate The approval of the project by the ethics committee allowed obtaining verbal informed consent from the patient to participate in the study.
Consent for Publication The authors consent to publish the content of this original report.

Conflict of Interest The authors declare that they have no conflicts of interest.

\section{References}

1. Li Q, Guan X, Wu P, Wang X, Zhou L, Tong Y, et al. Early transmission dynamics in Wuhan, China, of novel coronavirus-infected pneumonia. N Engl J Med. 2020;382:1199-207.

2. Huang C, Wang Y, Li X, Ren L, Zhao J, Hu Y, et al. Clinical features of patients infected with 2019 novel coronavirus in Wuhan. China Lancet. 2020;395:497-506.

3. Brodin P. Immune determinants of COVID-19 disease presentation and severity. Nat Med. 2021;27:28-33.

4. Carfî A, Bernabei R, Landi F. for the Gemelli Against COVID-19 Post-Acute Care Study Group. Persistent Symptoms in patients after acute COVID-19. JAMA. 2020;324:603-5.

5. Garrigues E, Janvier P, Kherabi Y, Le Bot A, Hamon A, Gouze $\mathrm{H}$, et al. Post-discharge persistent symptoms and health-related quality of life after hospitalization for COVID-19. J Infect. 2020;81:E4-6.

6. Zhou Y, Fu B, Zheng X, Wang D, Zhao C, qi Y, et al. Pathogenic T-cells and inflammatory monocytes incite inflammatory storms in severe COVID-19 patients. Natl Sci Rev. 2020;7:998-1002.

7. Masiá M, Fernández-González M, Padilla S, Ortega P, García JA, Agulló V, et al. Impact of interleukin-6 blockade with tocilizumab on SARS-CoV-2 viral kinetics and antibody responses in patients with COVID-19: A prospective cohort study. EBioMedicine 2020; 60:102999.

8. Nalbandian A, Sehgal K, Gupta A, Madhavan MV, McGroder C, Stevens JS, et al. Post-acute COVID-19 syndrome. Nat Med. 2021;27:601-15.

9. Common Investigation Protocol for Investigating Suspected SARS-CoV-2 Reinfection. Centers for Disease Control and Prevention. Available at: https://www.cdc.gov/coronavirus/2019ncov/php/reinfection.html. [Accessed 4 March 2021].

10. Long QX, Tang XJ, Shi QL, Li Q, Deng HJ, Yuan J, et al. Clinical and immunological assessment of asymptomatic SARS-CoV-2 infections. Nat Med. 2020;26:1200-4.

11. Ibarrondo FJ, Fulcher JA, Goodman-Meza D, Elliott J, Hofmann C, Hausner MA, et al. Rapid decay of anti-SARS-CoV-2 antibodies in persons with mild COVID-19. N Engl J Med. 2020;383:1085-7.

12. Duysburgh E, Mortgat L, Barbezange C, Dierick K, Fischer N, Heyndrickx L, et al. Persistence of IgG response to SARS-CoV-2. Lancet Infect Dis. 2021;21:163-4.

13. Seow J, Graham C, Merrick B, Acors S, Pickering S, Steel KJA, et al. Longitudinal observation and decline of neutralizing antibody responses in the three months following SARS-CoV-2 infection in humans. Nature Microbiol. 2020;5:1598-607.

14. Huang C, Huang L, Wang Y, Li X, Ren L, Gu X, et al. 6-month consequences of COVID-19 in patients discharged from hospital: a cohort study. Lancet. 2021;397:220-32.

15. Cao WC, Liu W, Zhang PH, Zhang F, Richardus JH. Disappearance of antibodies to SARS-associated coronavirus after recovery. N Engl J Med. 2007;357:1162-3.

16. Forthal DN. Functions of Antibodies Microbiol Spectr. 2014;2:1-17.

17. Tsuboi N, Asano K, Lauterbach M, Mayadas TN. Human neutrophil Fcgamma receptors initiate and play specialized 
nonredundant roles in antibody-mediated inflammatory diseases. Immunity. 2008;28:833-46.

18. Bunk S, Sigel S, Metzdorf D, Sharif O, Triantafilou K, Triantafilou $\mathrm{M}$, et al. Internalization and coreceptor expression are critical for TLR2-mediated recognition of lipoteichoic acid in human peripheral blood. J Immunol. 2010;185:3708-17.

19. Fernandez N, Renedo M, Alonso S, Crespo MS. Release of arachidonic acid by stimulation of opsonic receptors in human monocytes: the FcgammaR and the complement receptor 3 pathways. J Biol Chem. 2003;278:52179-87.

20. Wagner E, Frank MM. Therapeutic potential of complement modulation. Nat Rev Drug Discov. 2010;9:43-56.

21. Rojas M, Rodríguez Y, Monsalve DM, Acosta-Ampudia Y, Camacho B, Gallo JE, et al. Convalescent plasma in Covid-19: possible mechanisms of action. Autoimmun Rev 2020; 19:102554.

22. Joyner MJ, Carter RE, Senefeld JW, Klassen SA, Mills JR, Johnson PW, et al. Convalescent plasma antibody levels and the risk of death from Covid-19. N Engl J Med 13 Jan 2021; NEJMoa2031893. Available at: https://doi.org/10.1056/NEJMoa2031893.

23. Weinreich DM, Sivapalasingam S, Norton T, Ali S, Gao H, Bhore $\mathrm{R}$, et al. REGN-COV2, a neutralizing antibody cocktail, in outpatients with Covid-19. N Engl J Med. 2021;384:238-51.

24. Garcia-Beltran WF, Lam EC, Astudillo MG, Yang D, Miller TE, Feldman J, et al. COVID-19-neutralizing antibodies predict disease severity and survival. Cell. 2021;184:476-88.

25. Rodriguez LST, Pou C, Lakshmikanth T, Zhang J, Mugabo CH, Wang $\mathrm{J}$, et al. Achieving symptom relief in patients with myalgic encephalomyelitis by targeting the neuro-immune interface and inducing disease tolerance. BioRxiv [Preprint]. February 28, 2020. Available at https://doi.org/10.1101/2020.02.20.958249.

26. Blundell S, Ray KK, Buckland M, White PD. Chronic fatigue syndrome and circulating cytokines: a systematic review. Brain Behav Immun. 2015;50:186-95.

27. Guillot X, Ribera A, Gasque P. Chikungunya-induced arthritis in Reunion Island: a long-term observational follow-up study showing frequently persistent joint symptoms, some cases of persistent chikungunya immunoglobulin $\mathrm{M}$ positivity, and no anticyclic citrullinated peptide seroconversion after 13 years. J Infect Dis. 2020;222:1740-4.

28. Lee AM, Wong JG, McAlonan GM, Cheung V, Cheung C, Sham PC, et al. Stress and psychological distress among SARS survivors 1 year after the outbreak. Can J Psychiatry. 2007;52:233-40.

29. Halpin SJ, McIvor C, Whyatt G, Adams A, Harvey O, McLean L, et al. Postdischarge symptoms and rehabilitation needs in survivors of COVID-19 infection: A cross-sectional evaluation. J Med Virol. 2021;93:1013-22.

30. Hall J, Myall K, Lam JL, Mason T, Mukherjee B, West A, et al. Identifying patients at risk of post-discharge complications related to COVID-19 infection. Thorax 2021; Feb 4: thoraxjnl-2020-215861.

31. Hosey MM, Needham DM. Survivorship after COVID-19 ICU stay. Nat Rev Dis Primers. 2020;6:60.

Publisher's Note Springer Nature remains neutral with regard to jurisdictional claims in published maps and institutional affiliations. 